\title{
PLANT AND CONTROL DESIGN USING CONVEXIFYING LMI METHODS
}

\author{
J. F. Camino, M. C. de Oliveira, and R. E. Skelton
}

Dept. of Mechanical and Aerospace Engrg., UC San Diego, La Jolla, CA, 93092-0411

\begin{abstract}
This paper presents a methodology in the Linear Matrix Inequality (LMI) framework to jointly optimize the linear control law and the linear parameters in the plant. The paper solves an integrated plant and control design problem which bounds the covariance of selected outputs. The method simultaneously designs the plant parameters and the controller. The proposed method also allows one to guarantee bounds on the peak response in the presence of bounded energy excitations. With minor modifications, the method can also guarantee bounds on the $H_{\infty}$ performance and many other convex performance criteria. The nonconvex problem is approximated by a convex one by adding a certain function to make the constraint convex. This "convexifying" function is updated with each iteration until the added convexifying function disappears at a saddle point of the nonconvex problem. Copyright $\odot 2002$ IFAC
\end{abstract}

Keywords: Plant and control design, hybrid control, LMI, convex performance.

\section{INTRODUCTION}

During the last two decades, the mathematical tools of control theory have produced algorithms which allow one to bound the dynamic response, given a class of uncertain time varying disturbances. Under some mild assumptions, such tools can also be used for plant design, even if no control is involved. In this context our approach for structure design allows performance bounds on the dynamic response of the output, whereas the more standard structural design code focus on the static response and eigenvalues.

The plant design of concern to us is designing the structure parameters mass, stiffness, and damping of a mechanical system. Thus, we focus on plants described in the form $E \dot{x}=A x+B_{u} u+B_{w} w$. Mechanical systems naturally appears in this form where $E$ contains a mass matrix which depends affinely on some free parameters.

It is a well known fact that the design of the plant and the design of a controller for a given system are not independent. In fact, the simultaneous design of structure and controller is a nonconvex optimization problem and is not in practice tractable. The available algorithms are computationally expensive (see Grandhi (1989); Jin and Sepulveda (1995); Onoda and Haftka (1987), and the references therein), without guaranteeing even a local minimum. It can be shown that the integrated plant and control design problem is equivalent to a decentralized output feedback control problem, which is well known to be hard to solve.

Following a two step redesign approach, one idea extensively used by Grigoriadis et al. (1996) was as follows. In the first step, a controller for a given nominal plant was designed to meet some prescribed closed loop performance bound $\gamma$. In a second step, the plant and the controller were simultaneously redesigned in order to minimize the active control energy subject to the constraint that the closed loop system matrix is kept constant. This is the "convexifying" constraint, but this constraint modifies the necessary conditions for a local optimum, so even a local optimum cannot be guaranteed for the original problem. A further improvement was given in Lu and Skelton (2000), 
where the authors considered more general structures and used the mixed $\mathrm{H}_{2} / \mathrm{H}_{\infty}$ performance criteria via a LMI (Linear Matrix Inequality) framework, but in the redesign step they still needed the convexifying constraint of matching the system matrix. A more direct LMI approach that does not impose constraints in the closed loop system matrices was used in Grigoriadis and Skelton (1998), but instead constraint the covariance matrix. This convexifying constraint again prevents the guarantee of a local minimum. The techniques solves the two step redesign procedure by iterating between two convex LMI subproblems. This approach has the drawback that it does not allow the mass matrix to be optimized.

Our paper presents a new theory for the simultaneous design of structure and controller that improves the existing methodologies in the following ways: Our approach is completely posed in the LMI framework, so many different type of convex performances and convex constraints can be incorporated; The method also allows one to optimize the mass parameter of the system. More precisely, it allows one to optimize any parameter that appears affinely in any of the system matrices.

\section{PROBLEM STATEMENT}

A large class of dynamic systems in the field of mechanics and structures can be represented by a vector second order differential equation of the form

$$
M \ddot{q}+D \dot{q}+S q=\hat{B}_{u} u(t)+\hat{B}_{w} w(t)
$$

where $q \in \mathbb{R}^{n}$ is the vector of generalized coordinates, $M \in \mathbb{R}^{n \times n}, S \in \mathbb{R}^{n \times n}$, and $D \in \mathbb{R}^{n \times n}$. The matrix $M$ is symmetric and positive definite. The control is $u(t)$, and the disturbance is $w(t)$. We cast our problem in the stochastic framework so that $w(t)$ is a white noise process.

Using a convenient change of variables given by $x=$ $\left[\begin{array}{ll}q^{T} & \dot{q}^{T}\end{array}\right]^{T}$, the second order differential equation (1) is promptly written in the representation

$$
\left[\begin{array}{cc}
I & 0 \\
0 & M
\end{array}\right] \dot{x}=\left[\begin{array}{cc}
0 & I \\
-S & -D
\end{array}\right] x+\left[\begin{array}{c}
0 \\
\hat{B}_{u}
\end{array}\right] u+\left[\begin{array}{c}
0 \\
\hat{B}_{w}
\end{array}\right] w
$$

or equivalently

$$
E \dot{x}=A x+B_{u} u+B_{w} w .
$$

In our approach for the integrated plant and control design problem, the parameters of the plant which are available for optimization appear affinely in the matrices $M, D$, and $S$. By this affine representation we mean that

$$
\begin{gathered}
M(\eta)=M_{0}+\sum_{s} \eta_{s} M_{s}, \quad D(\beta)=D_{0}+\sum_{j} \beta_{j} D_{j}, \\
S(\gamma)=S_{0}+\sum_{k} \gamma_{k} S_{k} .
\end{gathered}
$$

where $M_{s}, D_{j}$, and $S_{k}$ are given matrices. Since matrix $A$ in (2) is affine in $D$ and in $S$, and matrix $E$ is affine in $M$, the system (2) can also be written as

$$
E(\alpha) \dot{x}=A(\alpha) x+B_{u} u+B_{w}(\alpha) w,
$$

for $A(\alpha)$ and $E(\alpha)$ affine matrices given by

$$
\begin{gathered}
A(\alpha)=A_{0}+\sum_{i} \alpha_{i} A_{i}, \quad E(\alpha)=E_{0}+\sum_{i} \alpha_{i} E_{i}, \\
B_{w}(\alpha)=B_{w 0}+\sum_{i} \alpha_{i} B_{w i},
\end{gathered}
$$

where the variable $\alpha$ contains, in a convenient way, the variables $\eta, \beta$, and $\gamma$, i.e., $\alpha=(\eta, \beta, \gamma)$. We adopt the representation (2) in order to preserve the affine property of the matrix $M$.

\subsection{Integrated Structure/Control}

For simplicity of presentation, we assume full state feedback, with the control law given by $u(t)=K x(t)$. The derivation for the full-order output feedback case does not require much more sophistication (see the extended version Camino et al. (2001)). The output vector for performance evaluation is

$$
z(t)=C_{z} x(t) .
$$

We first present Theorem 1 which characterizes the control problem for the structure and control design, with a stochastic interpretation. The disturbance $w(t)$ applied to the system is assumed to be a stochastic white noise process with intensity $W=W^{T}>0$, i.e., $\mathscr{E}\left\{w(t) w(\tau)^{T}\right\}=W \delta(t-\tau)$, where $\mathscr{E}$ is the expectation operator. Our performance criteria is to minimize the variance of the control $u(t)$ applied to the system, while the output $z(t)$ is bounded in the sense

$$
\lim _{t \rightarrow \infty} \mathscr{E}\left\{z(t) z(t)^{T}\right\}<\Omega,
$$

for some given positive definite matrix $\Omega$.

Theorem 1. Assume that $w(t)$ is a white noise process with intensity $W=W^{T}>0$. Define $F=K P$. Let $\gamma>0$ be a given scalar, $\Omega$ be a given positive definite matrix, and consider the system (2). Then the following statements are equivalent:

(i) There exists parameter $\alpha$, and a stabilizing state feedback $u(t)=K x(t)$ such that

$$
\lim _{t \rightarrow \infty} \mathscr{E}\left\{z(t) z(t)^{T}\right\}<\Omega
$$

and

$$
\lim _{t \rightarrow \infty} \mathscr{E}\left\{u(t)^{T} u(t)\right\}<\gamma
$$

(ii) There exists matrices of compatible dimensions $P=P^{T}>0, U=U^{T}>0$, and $F$, and parameter $\alpha$, such that the following inequalities are satisfied

$$
\left[\begin{array}{cc}
(*) & (\bullet)^{T} \\
B_{w}(\alpha)^{T} & -W^{-1}
\end{array}\right]<0
$$




$$
\operatorname{Tr}(U)<\gamma, \quad\left[\begin{array}{cc}
U & F \\
F^{T} & P
\end{array}\right]>0, \quad C_{z} P C_{z}^{T}<\Omega .
$$

where $(*)$ refers to $B_{u} F E(\alpha)+E(\alpha) F^{T} B_{u}^{T}+$ $A(\alpha) P E(\alpha)+E(\alpha) P A(\alpha)^{T}$.

(iii) For $G$ given by $G=\left(A+B_{u} K-E\right) P$, there exists matrices of compatible dimensions $Q=Q^{T}>0$, $U=U^{T}>0$, and $K$, and parameter $\alpha$, such that

$$
\begin{aligned}
& {\left[\begin{array}{cccc}
(*) & (\bullet)^{T} & (\bullet)^{T} & (\bullet)^{T} \\
B_{w}(\alpha)^{T} & -W^{-1} & (\bullet)^{T} & (\bullet)^{T} \\
A(\alpha)^{T}+K^{T} B_{u}^{T} & 0 & -Q & (\bullet)^{T} \\
E(\alpha)^{T} & 0 & 0 & -Q
\end{array}\right]<0,} \\
& \operatorname{Tr}(U)<\gamma, \quad\left[\begin{array}{cc}
U & K \\
K^{T} & Q
\end{array}\right]>0, \quad\left[\begin{array}{cc}
\Omega & C_{z} \\
C_{z}^{T} & Q
\end{array}\right]>0 .
\end{aligned}
$$

where $(*)$ refers to the term $G Q G^{T}-(A(\alpha)+$ $\left.B_{u} K-E(\alpha)\right) G^{T}-G\left(A(\alpha)+B_{u} K-E(\alpha)\right)^{T}$.

We present the proof of this theorem in Section 3.1, after the necessary tools provided by the convexifying algorithm have been introduced in Section 3 .

If the matrices $A$ and $E$ do not depend on the structure parameter $\alpha$, then the constraint (5) in item (ii) is an LMI, hence a convex set, in $U, P$, and $F$. In other words, if the structure is known, the problem reduces to a standard convex state feedback control problem. If the matrices $A$ and $E$ depend on $\alpha$, then the product $A(\alpha) P E(\alpha)+B_{u} F E(\alpha)$ is nonlinear in the decision variables $\alpha, F$, and $P$. In this case, it is hard to find a solution.

Note that independently of the control parameter $K$, the product of the system matrix $A(\alpha)$ and the "Lyapunov" matrix $P$ is always present in (5). When the mass matrix $M$ is fixed (matrix $E$ does not depend on the parameter $\alpha$ ), the procedure adopted in Grigoriadis and Skelton (1998) is to iterate between two convex subproblems: first, for fixed structure parameter $\alpha$ solve for the Lyapunov matrix $P$ in (5); second, for fixed matrix $P$ solve for the parameter of the structure $\alpha$ in (5). This strategy in practice converges to a solution, although there are no guarantee, even for a local minimum.

The algorithm we propose in this paper also iterates between two subproblems, but in a more elaborate way. Before iterating, we apply some convexifying potential functions to the nonconvex constraint in order to generate the conditions in item (iii). Notice that, for given matrix $G$, these conditions are simultaneously affine in the variables $Q, U, K$ and $\alpha$. In this sense the joint structure/control problem has been "convexified". Therefore, there will be no need to fix the Lyapunov matrix $P$ in the redesign step (instead, the fixed matrix will be the added potential matrix $G$ ). The convexifying potential method and the algorithm will be detailed in Section 3 .

\section{THE CONVEXIFYING ALGORITHM}

In this section we shall present the theory behind the convexifying algorithm, which is a powerful tool for solving control problems with structure imposed on the controller. For a detailed presentation of the convexifying potential functions applied to control theory, see de Olieveira et al. (2000). In the integrated design problem stated herein, we do not impose constraints on the control gain matrix, although the control law could be subject to arbitrary affine structural constraints, enabling one to solve complex joint structure/control design problems. However, it is possible to show that the free structural parameters create the equivalence of a decentralized control problem where the "control" gain matrix is diagonal.

In the sequel, we propose an algorithm to find stationary solutions to the nonconvex structure/control problem using the idea of the convexifying potential functions developed in de Olieveira et al. (2000). In that paper, it was shown that many standard control problems such as $H_{2}$ and $H_{\infty}$ problems with some imposed structure in the controller can be formulated as an LMI problem having an extra nonconvex constraint. To use convex tools, convexifying potential functions were added to the nonconvex constraint. A convexifying algorithm to solve this nonconvex problem was also proposed. This algorithm iterates in a sequence of convex subproblems which, given some conditions, is guaranteed to converge to a stationary point of the original nonconvex problem.

Let $x, \eta$ belong to a convex set $\phi$, and $\mathscr{F}(x)$ be a nonconvex matrix function. A convexifying potential function is a differentiable function $\mathscr{G}(x, \eta)$ such that the function $\mathscr{F}(x)+\mathscr{G}(x, \eta)$ for all $x, \eta \in \phi$ is convex in $x$. Thus, if $\mathscr{F}(x)$ satisfies certain conditions, a stationary point of the nonconvex optimization problem

$$
\bar{x}=\arg \min _{x \in \Omega} f(x), \Omega=\{x \in \phi \mid \mathscr{F}(x)<0\}
$$

can be obtained by iterating on a sequence of convex subproblems given by

$$
\begin{aligned}
x_{k+1}= & \arg \min _{x \in \Omega_{k}} f(x), \\
& \Omega_{k}=\left\{x \in \phi \mid \mathscr{F}(x)+\mathscr{G}\left(x, x_{k}\right)<0\right\} .
\end{aligned}
$$

To impose that the optimality conditions of both optimization problems (9) and (10) are identical, the potential function $\mathscr{G}$ must possess some extras properties such as being nonnegative definite and that $\mathscr{G}(x, \eta)=$ 0 if, and only if, $x=\eta$.

In order to apply the above idea to the integrated structure and control design, we shall define the nonconvex function $\mathscr{F}$ of interest. From the set of conditions (ii) given in Theorem 1 we have that the constraint with nonlinear terms is (5). Completing the squares, this inequality can be manipulated into 


$$
\begin{aligned}
0> & {\left[\begin{array}{cc}
A_{c l} P E+E P A_{c l}^{T} & (\bullet)^{T} \\
B_{w}^{T} & -W^{-1}
\end{array}\right]=} \\
& {\left[\begin{array}{cc}
\left\{\begin{array}{c}
A_{c l} P A_{c l}^{T}+E P E^{T}- \\
\left(A_{c l}-E\right) P\left(A_{c l}-E\right)^{T}
\end{array}\right\} & B_{w} \\
B_{w}^{T} & -W^{-1}
\end{array}\right] }
\end{aligned}
$$

where $A_{c l}(\alpha, K)=A(\alpha)+B_{u} K$. The dependence of the matrices $A_{c l}, B_{w}$, and $E$ on $\alpha$ and $K$ is suppressed to simplify the notation.

Let $x:=(P, \alpha, K)$ and define $Q=P^{-1}$, then by applying Schur complements we can rewrite the above inequality $\mathscr{F}(x)$ into the equivalent form

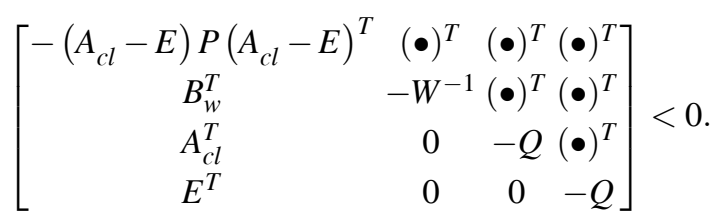

Using this concave inequality to define $\mathscr{F}$ it will be possible to guarantee convergence of the convexifying algorithm to a saddle point of the original nonconvex problem.

Now, we shall define a potential convexifying function $\mathscr{G}$ that makes $\mathscr{F}+\mathscr{G}$ convex. For this purpose, let $G$ be given by

$$
G(x):=\left(A_{c l}-E\right) P,
$$

and define the convexifying potential function $\mathscr{G}(x, \eta)$ for the above nonconvex matrix function $\mathscr{F}$ to be the nonnegative definite expression given by

$$
\begin{aligned}
& \mathscr{G}(x, \eta):= \\
& \left(A_{c l}-E-G(\eta) P^{-1}\right) P\left(A_{c l}-E-G(\eta) P^{-1}\right)^{T} .
\end{aligned}
$$

This function satisfies the convexifying assumptions, since it is nonnegative definite and (12) attains zero for the choice of matrices $x=\eta$, that is, $\mathscr{G}(x, \eta)=0$ for $x=\eta$. Moreover, adding (12) to the first block of (11), we obtain the following LMI in $Q$ and $K$

$$
\left[\begin{array}{cccc}
(*) & (\bullet)^{T} & (\bullet)^{T} & (\bullet)^{T} \\
B_{w}^{T} & -W^{-1} & (\bullet)^{T} & (\bullet)^{T} \\
A_{c l}^{T} & 0 & -Q & (\bullet)^{T} \\
E^{T} & 0 & 0 & -Q
\end{array}\right]<0 .
$$

where $(*)$ refers to the term

$$
-\left(A_{c l}-E\right) G^{T}-G\left(A_{c l}-E\right)^{T}+G Q G^{T} .
$$

The dependence of $G$ on $\eta$ has been also suppressed for simplicity. Substituting $A_{c l}=A(\alpha)+B_{u} K$ into the above inequality one recover inequality (7) given in Theorem 1. In this form, the Lyapunov matrix $P=Q^{-1}$ and the system matrices $A(\alpha)$ and $E(\alpha)$ no longer appear as products. Instead, these products have being replaced by products with $G(\eta)$, which has been introduced with the potential function. Notice that $\eta$ is kept constant and equal to $\eta=x_{k}$ in the convex subproblems to be solved of the form (10).
Considering as the objective function to be minimized an upper bound on the covariance of the control energy, that is, $f=\gamma>\mathscr{E}\left\{u(t)^{T} u(t)\right\}$, the ideas explained so far are summarized in the algorithm below.

\section{Convexifying Algorithm for Structural Con- trol - CASC}

Let $f=\min \gamma$.

Set the nominal values for $\alpha_{0}, A_{0}$, and $E_{0}$.

Compute $K_{0}$ and $P_{0}$ by finding a feasible solution to item (ii) of Theorem 1.

Set $\varepsilon$ to some prescribed tolerance and $k=0$.

\section{Repeat}

Set $G_{k} \leftarrow\left(A_{c l}^{k}\left(\alpha_{k}\right)-E\left(\alpha_{k}\right)\right) P_{k}$.

For fixed $G=G_{k}$, solve $f_{k}=\min \gamma$ subject to (7-8) for $\alpha, K, Q$, and $U$.

Denote the solution $\left(\alpha^{*}, K^{*}, Q^{*}, U^{*}\right)$.

Set $\left(P_{k+1}, \alpha_{k+1}, K_{k+1}\right) \leftarrow\left(Q^{*-1}, \alpha^{*}, K^{*}\right)$.

$k \leftarrow k+1$.

Until $\left\|f_{k}-f_{k-1}\right\|<\varepsilon$

\subsection{Proof of Theorem 1}

Proof: The discussion in the previous section can be used to show the equivalence between conditions (ii) and (iii) given in Theorem 1. If the constraints in (ii) have a feasible solution $\bar{x}:=\left(\bar{P}, \bar{\alpha}, \bar{F} \bar{P}^{-1}\right)$, then $\mathscr{F}(\bar{x})<0$. Hence the constraints in (iii) also have a feasible solution (with $G(\bar{x}):=\left(A_{c l}(\bar{\alpha})-E(\bar{\alpha})\right) \bar{P}$ ) since $\mathscr{G}(\bar{x}, \bar{x})=0$ by definition of the convexifying function. Conversely, if the constraints (iii) have a feasible solution $\bar{x}, \bar{\eta}$, then $\mathscr{F}(\bar{x})+\mathscr{G}(\bar{x}, \bar{\eta})<0$. But since by definition $\mathscr{G}(\bar{x}, \bar{\eta}) \geq 0$, we have that $\mathscr{F}(\bar{x}) \leq$ $\mathscr{F}(\bar{x})+\mathscr{G}(\bar{x}, \bar{\eta})<0$, which implies that the constraints in $(i i)$ are also feasible.

The equivalence between $(i)$ and (ii) in Theorem 1 is provided by the following argument. Since, by assumption, matrix $M(\alpha)$ is positive definite for all $\alpha$ of interest, the matrix $E(\alpha)$ is invertible. Hence, for the state feedback law given by $u(t)=K x$, the closed loop system

$$
E(\alpha) \dot{x}=A_{c l}(\alpha) x+B_{w}(\alpha) w .
$$

can be equivalently written in standard state space form as

$$
\begin{aligned}
\dot{x} & =E(\alpha)^{-1}\left(A_{c l}(\alpha) x+B_{w}(\alpha) w\right) \\
& =\bar{A}_{c l}(\alpha) x+\bar{B}_{w}(\alpha) w .
\end{aligned}
$$

For the above stable state space system it is a standard result (Skelton et al. (1998)) that the conditions in item (i) of Theorem 1 hold if, and only if

$$
\mathscr{E}\left\{u(t)^{T} u(t)\right\}=\operatorname{Tr}\left(K P K^{T}\right)<\gamma
$$

and

$$
\mathscr{E}\left\{z(t) z(t)^{T}\right\}=C_{z} P C_{z}^{T}<\Omega,
$$

where the matrix $P=P^{T}>0$ satisfies

$$
\bar{A}_{c l}(\alpha) P+P \bar{A}_{c l}(\alpha)^{T}+\bar{B}_{w}(\alpha) W \bar{B}_{w}(\alpha)^{T}<0 .
$$


Applying a congruence transformation by multiplying on the left and on the right side by the symmetric matrix $E(\alpha)$, we obtain the equivalent inequality

$$
\begin{aligned}
& A_{c l}(\alpha) P E(\alpha)+E(\alpha) P A_{c l}(\alpha)^{T}+ \\
& B_{w}(\alpha) W B_{w}(\alpha)^{T}<0 .
\end{aligned}
$$

Using a Schur complement, it is possible to show that the above inequality is equivalent to

$$
\left[\begin{array}{cc}
A_{c l}(\alpha) P E(\alpha)+E(\alpha) P A_{c l}(\alpha)^{T} & B_{w}(\alpha) \\
B_{w}(\alpha)^{T} & -W^{-1}
\end{array}\right]<0 .
$$

Noting that $A_{c l}(\alpha)=A(\alpha)+B_{u} K$ and $F=K P$, inequality (13) becomes inequality (5) given in (ii). To show (6) we introduce the auxiliary symmetric variable $U$ such that

$$
U>K P K^{T}=F P^{-1} F^{T}
$$

then $\gamma>\operatorname{Tr}(U)>\operatorname{Tr}\left(K P K^{T}\right)$. Hence, using Schur complement, this inequality is equivalent to

$$
\operatorname{Tr}(U)<\gamma, \quad\left[\begin{array}{cc}
U & F \\
F^{T} & P
\end{array}\right]>0 .
$$

This completes the proof.

\section{EXAMPLES}

To illustrate the proposed methodology, we choose the problem of isolating a civil engineering structure against earthquakes (Kose et al. (1998); Ramalho et al. (2000); Camino et al. (2001)).

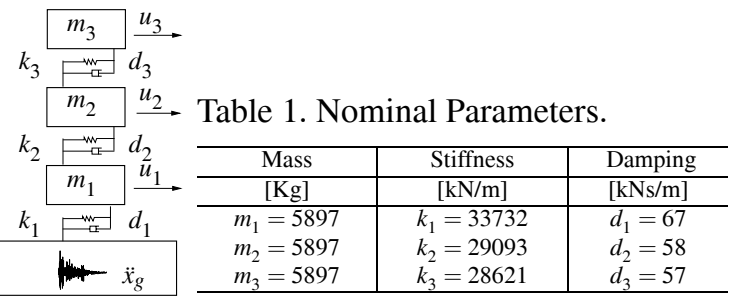

Fig. 1. Model

The model of the system in consideration is shown in Figure 1, and the nominal values are given in Table 1 . The control inputs $u_{i}$ are independent forces applied to each floor. Hence, for the model (2), $\hat{B}_{u}=I$, the matrix $\hat{B}_{w}$ is given by $\hat{B}_{w}=\left(m_{1}, m_{2}, m_{3}\right)^{T}$, and the disturbance vector $w$ is assumed to be a white noise process with intensity $W=16 \mathrm{~m}^{2} / \mathrm{s}^{4}$, which represents the earthquake acceleration of the ground motion $\ddot{x}_{g}$ in Figure 1 .

For this dynamic system (2), the mass matrix is given by $M=\operatorname{diag}\left(m_{1}, m_{2}, m_{3}\right)$, and the stiffness matrix $S$, and the damping matrix $D$ are given by:

$$
\begin{aligned}
S=\left[\begin{array}{ccc}
k_{1}+k_{2} & -k_{2} & 0 \\
-k_{2} & k_{2}+k_{3} & -k_{3} \\
0 & -k_{3} & k_{3}
\end{array}\right], \\
D=\left[\begin{array}{ccc}
d_{1}+d_{2} & -d_{2} & 0 \\
-d_{2} & d_{2}+d_{3} & -d_{3} \\
0 & -d_{3} & d_{3}
\end{array}\right]
\end{aligned}
$$

The states are the displacement and the velocity of each floor relative to the ground, i.e.: $q_{i}$ represents the displacement of the mass $m_{i}$, and $\dot{q}_{i}$ its velocity. It is assumed that the lower and the upper bounds on all the parameters are 0.5 and 2.0 of the nominal values in Table 1.

For this problem, we seek designs which limit the variance of the inter-story drift $z_{1}=q_{1}, z_{i+1}=q_{i+1}-$ $q_{i}, i=1,2$. Thus the output $C_{z}$ is given by

$$
C_{z}=\left[\begin{array}{cccccc}
1 & 0 & 0 & 0 & 0 & 0 \\
-1 & 1 & 0 & 0 & 0 & 0 \\
0 & -1 & 1 & 0 & 0 & 0
\end{array}\right]
$$

The imposed bounds in the inter-story drift are $\sqrt{\mathscr{E} z_{i}^{2}}$ $\leq 6.782 \times 10^{-3} \mathrm{~m}$. Thus, $\Omega$ is given by $\Omega=\operatorname{diag}(4.6$, $4.6,4.6) \times 10^{-5} \mathrm{~m}^{2}$.

We simulate three different cases of the integrated plant/control desgin. In the first case, all the parameters of the structure are optimized and the CASC algorithm converged after 176 iterations to the passive $\left(\mathscr{E}\left\{u^{T} u\right\}=0\right)$ solution:

\begin{tabular}{c|c|c} 
Mass & Stiffness & Damping \\
\hline$[\mathrm{Kg}]$ & {$[\mathrm{kN} / \mathrm{m}]$} & {$[\mathrm{kNs} / \mathrm{m}]$} \\
\hline$m_{1}^{*}=2948$ & $k_{1}^{*}=67463$ & $d_{1}^{*}=134$ \\
$m_{2}^{*}=2948$ & $k_{2}^{*}=55063$ & $d_{2}^{*}=116$ \\
$m_{3}^{*}=2948$ & $k_{3}^{*}=32699$ & $d_{3}^{*}=114$
\end{tabular}

The achieved output performance was binding, i.e., $\left[C_{z} P C_{z}^{T}\right]_{i i}=\Omega_{i i}$. The results are presented in Figure 2, where the width of the bar graphs are proportional

\begin{tabular}{|c|c|c|c|}
\hline Mass & Damping & Stiffness & Control \\
\hline 3 & & & $\mathscr{E}\left\{u^{T} u\right\}=0$ \\
\hline 2 & & & \\
\hline 1 & & & \\
\hline
\end{tabular}
to the amount of resources used (mass, damping, stiffness, and control). The dotted lines are the original structure and the shaded bar graphs show the final designs. This problem provides zero control when the inter-story drift is larger than $6.782 \times 10^{-3} \mathrm{~m}$, and provides active control energy for any bound smaller than $6.782 \times 10^{-3} \mathrm{~m}$.

Fig. 2. Designing $k_{1}, k_{2}, k_{3}, d_{1}, d_{2}, d_{3}, m_{1}, m_{2}, m_{3}$.

In the second case, only the structural parameters of the third floor are available for optimization, that is, $k_{3}, d_{3}$, and $m_{3}$. The CASC algorithm converged after 84 iterations to the solution:

\begin{tabular}{c|c|c} 
Mass & Stiffness & Damping \\
\hline$[\mathrm{Kg}]$ & {$[\mathrm{kN} / \mathrm{m}]$} & {$[\mathrm{kNs} / \mathrm{m}]$} \\
\hline$m_{3}^{*}=2948$ & $k_{3}^{*}=14311$ & $d_{3}^{*}=69$
\end{tabular}

with the control gain $K^{*}\left[\times 10^{3}\right]$ given by

$\left[\begin{array}{rrrrrr}-1793 & -2823 & -201 & -73 & -34 & -10 \\ 2023 & -1386 & -1363 & -34 & -73 & -30 \\ -27 & 1165 & -1243 & -20 & -61 & -55\end{array}\right]$.


This gain provides a control effort of $\sqrt{\mathscr{E}\left\{u^{T} u\right\}}=$ $\sqrt{14248721953} \mathrm{~N}$. The achieved output performance was $\left[C_{z} P C_{z}^{T}\right]_{i i}=(4.6,3.9,4.2) \times 10^{-5} \mathrm{~m}^{2}$. Note that the achieved displacement for the first floor was binding. The results are presented in Figure 3.

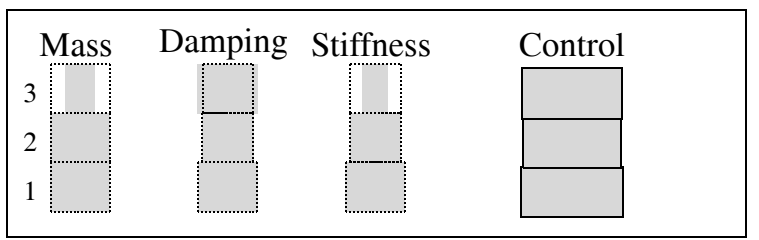

Fig. 3. Designing $k_{3}, d_{3}, m_{3}$.

In the third case, the structure is fixed at the initial values, and using only active control we seek the same output performance as before. This is the classical approach to structural control where the structure is design first, them the control is applied. The results (Figure 4) show that if we are not free to modify the structure, then a large amount of control energy is required $\left(\sqrt{\mathscr{E}\left\{u^{T} u\right\}}=\sqrt{24462917207} \mathrm{~N}\right)$ to achieve the prescribed bound $\sqrt{\mathscr{E} z_{i}^{2}} \leq 6.782 \times 10^{-3} \mathrm{~m}$.

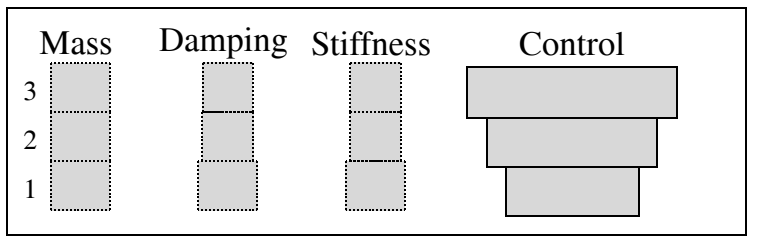

Fig. 4. State feedback with nominal structure.

This example shows that the control energy can be huge if the classical separation of the structure and control design is applied. In contrast, the control energy required to achieve the same objectives can be very small when some of the structural parameters are allowed to be jointly optimized with the controller.

\section{CONCLUSION}

This paper demonstrates the benefits of simultaneously designing the structure and the controller. The algorithm is proposed in the LMI framework, for which very efficient interior point methods are available. The design allows changes in any parameters that appear affinely in the system matrices. A stationary point of the nonconvex structure/control problem is found by a sequence of convex subproblems with the help of potential convexifying functions. The performance criteria used in the design was a bound on the output covariance of the closed loop system, but the methodology can incorporate many other convex criteria. The paper improves the techniques available in the literature in the sense that: the methodology is completely in the LMI framework, having no need to solve a constrained quadratic optimization problem; the technique allows parameters in the "descriptor" matrix $E$ to be optimized; and the proposed algorithm does not require that the Lyapunov matrix (which provides the control gain) to be fixed in the structure design step.

For a specified performance bound, the proposed CASC algorithm determines whether active feedback control is necessary or not. The problem minimizes the control energy required to achieve a specified bound $\Omega$ on the response. If the specified bound is large enough the algorithm produces a passive design (indicated by zero control energy in the optimized solution). If the required performance bound is small enough, no passive solution exists, and the algorithm produces a controller with minimal control energy to achieve the required performance $\Omega$.

\section{REFERENCES}

Camino, J. F., M. C. de Oliveira and R. E. Skelton (2001). Convexifying LMI methods for integrating structure and control design. Submitted to the ASCE Journal of Structural Engineering.

de Olieveira, M. C., J. F. Camino and R. E. Skelton (2000). A convexifying algorithm for the design of structured linear controller. In: Proceedings of the 39th IEEE Conference on Decision and Control. Sydney, Australia. pp. 2781-2786.

Grandhi, R. V. (1989). Structural and control optimization of space structures. Computers \& Structures 31(2), 139-150.

Grigoriadis, K. M. and R. E. Skelton (1998). Integrated structural and control design for vector second-order systems via LMIs. In: Proceedings of the American Control Conference. Philadelphia, Pennsylvania. pp. 1625-1629.

Grigoriadis, K. M., G. Zhu and R. E. Skelton (1996). Optimal redesign of linear systems. ASME Journal of Dynamic Systems, Measurements, and Control 118, 598-605.

Jin, Ik Min and Abdon E. Sepulveda (1995). Structural/control system optimization with variable actuator masses. AIAA Journal 33(9), 1709-1714.

Kose, I. E., F. Jabbari, W. E. Schmitendorf and J. N. Yang (1998). Controllers for quadratic stability and performance of a benchmark problem. Earthquake Engineering and Structural Dynamics 27(11), 1385-1397.

Lu, Jianbo and R. E. Skelton (2000). Integrating structure and control design to achieve mixed $\mathrm{H}_{2} / \mathrm{H}_{\infty}$ performance. Int. J. Control 73(16), 1449-1462.

Onoda, J. and R. T. Haftka (1987). An approach to structure/control simultaneous optimization for large flexible spacecraft. AIAA Journal 25(8), 1133-1139.

Ramalho, J. C., E. A. Johnson and B.F. Spencer (2000). "Smart" base isolation system. Submitted to the ACSE Journal of Engineering Mechanics.

Skelton, R. E., T. Iwasaki and K. M. Grigoriadis (1998). A Unified Approach to Control Design. Taylor and Francis. 